\title{
Bilateral retinopathy, aplastic anaemia, and central nervous system abnormalities: a new syndrome?
}

\author{
T Revesz, S Fletcher, L I Al-Gazali, P DeBuse
}

\section{Department of Paediatrics, Faculty of Medicine and Health Sciences, UAE University, PO Box 17666, Al Ain, United Arab Emirates. \\ T Revesz \\ L I Al-Gazali \\ $P$ DeBuse}

Department of Pathology, Tawam Hospital, PO Box 15258, Al Ain, UAE. $S$ Fletcher

Correspondence to Dr Revesz.

Received 28 November 1991. Revised version accepted 21 February 1992.

\begin{abstract}
A male infant was found to have bilateral exudative retinopathy at 6 months of age. A month later severe aplastic anaemia was diagnosed, eventually leading to the infant's death. Additional features of this seemingly new syndrome were intrauterine growth retardation, fine sparse hair, fine reticulate skin pigmentation, ataxia because of cerebellar hypoplasia, cerebral calcifications, extensor hypertonia, and progressive psychomotor retardation.
\end{abstract}

( $($ Med Genet 1992;29:673-5)

\section{Case report}

A 6 month old Sudanese male infant developed a squint and ophthalmic examination showed bilateral leucocoria. The mother was aged 30 years, gravida 6 , para 6 . The birth was by caesarean section for intrauterine growth retardation (birth weight $1800 \mathrm{~g}$ ) at term. No cause for the growth retardation was identified.

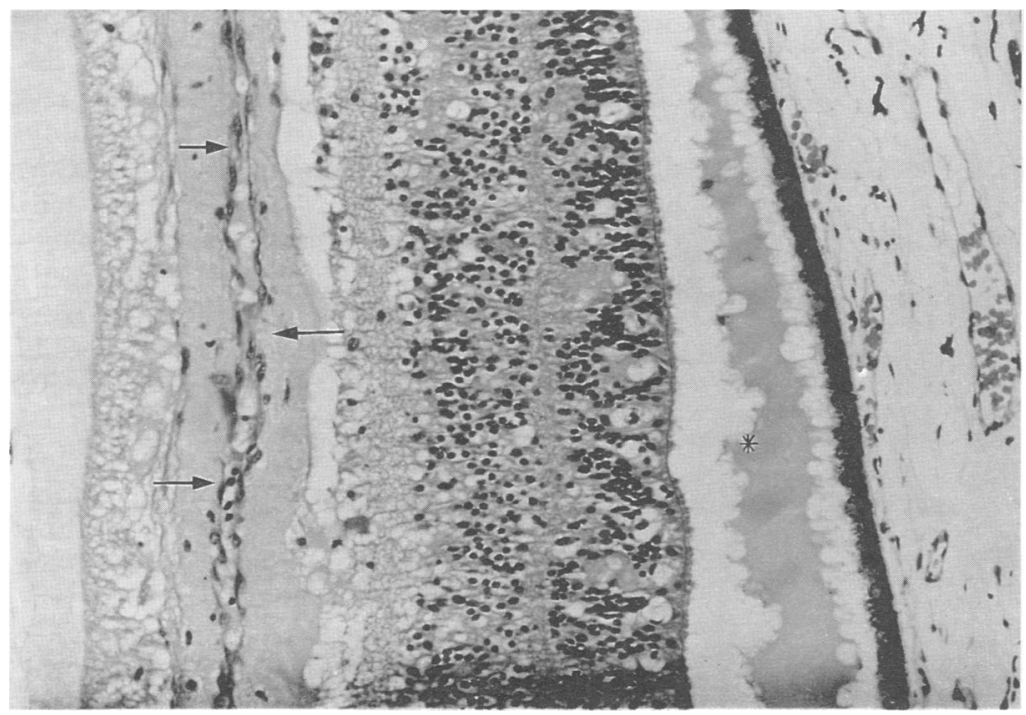

Figure 1 Section of the retina. The detachment of the retina with its contained fluid which separates the choroid from the array of torn retinal rods and cones is marked with an asterisk. The arrows point to a longitudinally sectioned retinal blood vessel. $A$ wide cuff of fluid exudate separates the endothelium from the surrounding connective tissue of the nerve layer ( $H \mathcal{E} E$ )
On first examination he was found to be well developed for his age. He had fine, sparse, straight hair, dark skin, and no obvious nail abnormalities. His skin showed some fine reticulate hyperpigmentation, mainly on his trunk and on his palms and soles. A $1 \times 1 \mathrm{~cm}$ purple patch was noted on the dorsum of his tongue, near the tip. Ear, nose, throat, heart, lungs, abdomen, and genitalia were all normal. $\mathrm{He}$ had bilateral leucocoria and roving nystagmus. $\mathrm{He}$ appeared to see only with peripheral vision. Tendon reflexes were brisk and muscle tone normal, but he had cervical and truncal ataxia. Social development seemed adequate.

Ophthalmological findings in the right eye were: megalocornea with slight buphthalmus; pupil unresponsive to light stimulation; whitish mass visible through the widely dilated pupil. The left eye showed similar but less severe changes. Fundoscopy showed whitegrey masses on both fundi originating from the retina. Orbital CT scan confirmed the presence of bilateral subretinal masses. Although no calcifications were seen inside the subretinal masses, retinoblastoma could not be ruled out.

Since no useful vision could be expected in this eye it was enucleated. Histologically, the enucleated eye showed no tumour but a prominent retinal detachment with a dense plasma exudate between the detached retina and the choroid was present. Over the detachment the retinal vessels were grossly abnormal, enlarged, and aneurysmal. They were expanded and filled with blood. The vessel walls were permeated by a pale eosinophilic plasma exudate that stripped off the endothelium which appeared now as a ring of cells in the lumen. The retina around the vessels was filled with a plasma exudate involving the nervous, ganglionic, and plexiform layers and irregularly penetrating the inner nuclear layer (fig 1). The ganglionic layer neurones were mostly non-viable. The appearances resembled Coats' retinopathy.

Soon after the enucleation anaemia and thrombocytopenia were noted. A bone marrow examination showed normocellular marrow with predominantly erythroid activity exhibiting megaloblastic changes. Megakaryocytes were noted as were other myeloid forms. Vitamin $B_{12}$ and folic acid levels were normal 
(232 pmol/1 and $36.5 \mathrm{nmol} / 1$, respectively). Erythropoietin level was also within the normal range $(786 \mathrm{mU} / \mathrm{ml})$. Metabolic studies, including plasma amino acids, lactate and pyruvate, urine mucopolysaccharides, orotic acid, and organic acids, were all within the normal range.

Over the next few weeks he became more pancytopenic and a repeat bone marrow examination showed severe hypoplasia and strands of fibrocollagenous tissue and fat, consistent with the diagnosis of severe aplastic anaemia. Hepatitis, HIV, parvovirus, CMV, and EBV antibodies were negative and no drugs or chemicals could be implicated in the aetiology of the aplasia. He did not have any of the characteristic features of Fanconi's anaemia and chromosome analysis showed a normal 46,XY karyotype. Chromosome fragility tests, however, could not be carried out. Short term bone marrow cultures showed no growth of colony forming units (granulocyte, macrophage, granulocyte-macrophage, or granulocytemacrophage-erythroid-megakaryocyte) and only one colony of burst forming unit (erythroid).

A CT scan of the brain showed widespread grey and white matter calcifications, consistent with but not typical of intrauterine infection There was also marked cerebellar hypoplasia and low attenuation changes in the white matter adjacent to the lateral ventricles (fig 2). Serological tests for toxoplasma, toxocara, rubella, and cysticercosis were all negative. CSF findings were normal.

The patient required regular packed cell and platelet transfusions. Corticosteroids, androgenic steroids, and granulocyte-macrophage colony stimulating factor treatment failed to

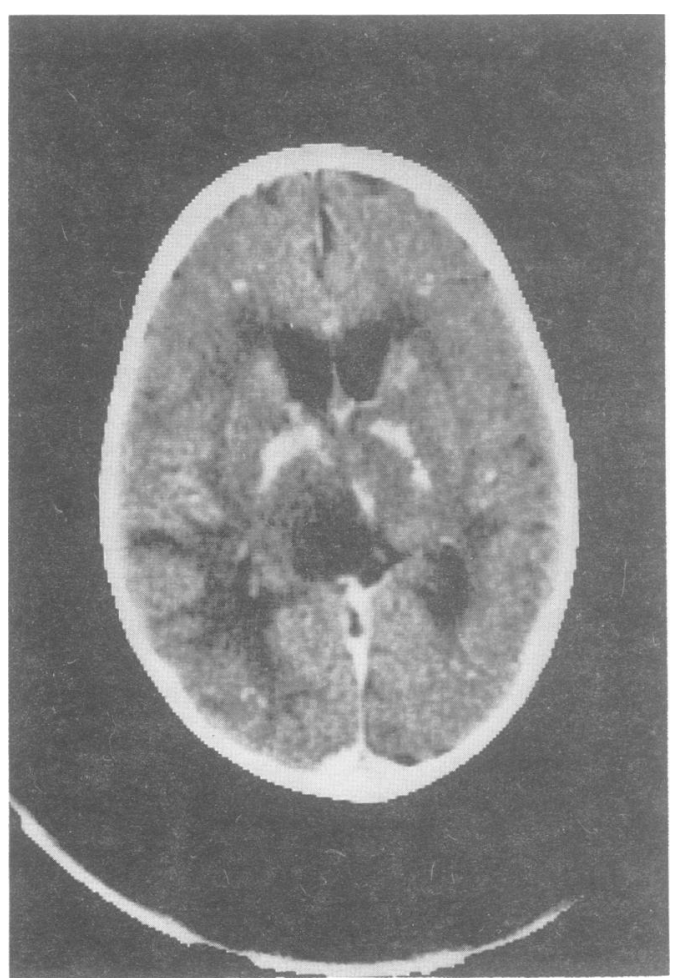

Figure $2 C T$ scan of the skull (post contrast image). Several calcifications are seen in the frontal periventricular white matter, the caudate nuclei, the internal capsule and thalamus, and the deep grey matter of the left temporal and both occipital lobes. elicit a response from the bone marrow. He also started to show signs of progressive neurological deterioration. Over the next few weeks he became less and less responsive to his mother, the irritability returned with some regularity, and he developed carpal spasms. Calcium, phosphorus, and magnesium levels were normal. His ataxia increased and he developed stridor with a hoarse cry. Lateral cervical spine $x$ ray showed a retropharyngeal mass. He was examined under anaesthesia and a compressible vascular swelling with the appearance of a haemangioma was found in the retropharynx at the cricoid level.

$\mathrm{He}$ continued to receive supportive treatment but his platelet count eventually became impossible to control with transfusions and the patient died at 19 months of age. In accordance with the parents' customs and wishes, no necropsy was performed.

\section{Discussion}

The case presented here clearly has many features that make it difficult to fit into any of the known disease entities. The severe vascular changes in the retina are similar to those of Coats' retinopathy. ${ }^{1}$ Tolmie et $a l^{2}$ reported a pair of sibs who showed many of the features that we encountered in our patient. In addition to the bilateral retinal changes all three patients had intrauterine growth retardation with resultant low birth weight, fine and somewhat sparse hair, and intracerebral calcifications. Chromosome studies in both the sisters and in our patient were normal. The sibs, however, had no haematological abnormalities.

In addition to the retinal exudation and detachment our patient developed severe haematological abnormalities that again do not seem to fit any of the well characterised diseases. The fine reticular pigmentation on his trunk and the darker than usual palms and soles raised the possibility of dyskeratosis congenita. Although the classical form of this disease often does not manifest until the second decade, several cases have been described in children. ${ }^{3}$ No specific laboratory test is known for dyskeratosis congenita. Diagnosis depends on the characteristic clinical triad of hyperpigmentation, nail dystrophy, and leucoplakia of the mucous membranes. The average age of the onset of symptoms seems to be around 7 to 10 years of age. ${ }^{4}$ Bone marrow failure usually develops in the second decade and those surviving into the third decade have a high chance of developing cancer. ${ }^{5}$

Our patient had what could be regarded as the earliest manifestations of the integumental changes of dyskeratosis congenita on his skin. Although he showed some features that have previously been described in dyskeratosis congenita, ${ }^{3}$ he did not have the typical skin, nail, and mucous membrane changes that are the hallmarks of this entity. Therefore, the diagnosis of dyskeratosis congenita was discounted.

The occurrence of retinal detachment, severe bone marrow aplasia, and progressive 
neurological abnormalities has not been described previously. We suggest this represents a hitherto unrecognised syndrome.

The authors wish to thank Professor T Gordon-Smith (St George's Hospital Medical School, London) for his valuable advice, and Drs P Swanberg, A Suvari, G Lestringent, and $\mathrm{Mr}$ P Fitzgerald for clinical and laboratory investigations.
1 Coats $\mathrm{G}$. Forms of retinal disease with massive exudation. $R$ Lond Ophthal Hosp Rep 1908;17:440-525.

2 Tolmie JL, Browne BH, McGettrick PM, Stephenson JBP. A familial syndrome with Coats' reaction retinal angiomas, hair and nail defects and intracranial calcification. Eye 1988;2:297-303.

3 Sirinavin C, Trowbridge AA. Dyskeratosis congenita: clinical features and genetic aspects. $\mathcal{f}$ Med Genet 1975;12:33954.

4 Davidson HR, Connor JM. Dyskeratosis congenita. 7 Med Genet 1998;25:843-6.

5 Friedland M, Lutton JD, Spitzer R, Levere RD. Dyskeratosis congenita with hypoplastic anemia: a stem cell defect. Am ₹ Hematol 1985;20:85-7. 